\title{
Missionplanning and Operational Constraints and their Resolution for EO Missions like EnMAP
}

\author{
Robert Axmann ${ }^{1}$ and Sabrina Eberle ${ }^{2}$ \\ German Space Operations Center \\ DLR Oberpfaffenhofen \\ 82230 Wessling, Germany
}

\begin{abstract}
Earth observation missions are generally operated by execution of predefined timetagged commands. However, depending on the type of mission, issues relevant to different types of mission may occur. These issues are drivers for the design of the operations system of EnMAP (Environmental Mapping and Analysis Program). The main challenges are limited transfer of telecommands and limited downlink capabilities; also the cloud coverage in the target area - for optical sensors - plays a huge role.
\end{abstract}

There are certain constraints, concerning this satellite bus in particular. For example a limited time tagged list which allows no more than 120 time tagged commands on board. Further the fact that every dumped house keeping packet has to be requested from the space craft which leads to a delay in the reception of telemetry can be a bottle neck. In addition to these S-band related issues it is a real challenge to coordinate the amount of payload data which will be down-linked via $\mathrm{X}$-band. In this paper the strategy of managing all these difficulties and optimally operating the satellite under these conditions, will be described.

For handling of cloud coverage a database with weather statistics will be implemented. These statistics are compared with global forecast models as provided by e.g. the German weather service (DWD). With the calculated forecast values, planning priority of datatakes is increased or decreased.

\section{Introduction}

$\mathrm{T}$ he German Space Operations Center (GSOC) is responsible for operations of different space missions. Operations experience dates back to the sixties, when the first satellites were operated from the control center. Meanwhile a fleet of missions with various sensors onboard is operated, beside completely scientific missions also technology demonstrations and military missions. Furthermore the European part of the ISS - the Columbus module - is operated.

Scientific mission portfolio operated by GSOC covers radar satellite missions like TDX \& TSX, technology missions like the up-coming TET, DEOS or OLEV as well as infrared missions BIRD or geo-science missions (CHAMP \& GRACE). EnMAP is intended to fill the gap in the area of mapping the Earth with a hyperspectral sensor. The mission is currently in preparation by German industry and the German aerospace agency. Once in orbit images of very high spectral resolution can be provided to the scientific community covering nearly each region on earth.

\footnotetext{
${ }_{1}^{1}$ Project Manager, Mission Operations, P.O. 1116, 82230 Wessling

${ }^{2}$ Project Manager, Mission Operations, P.O. 1116, 82230 Wessling 


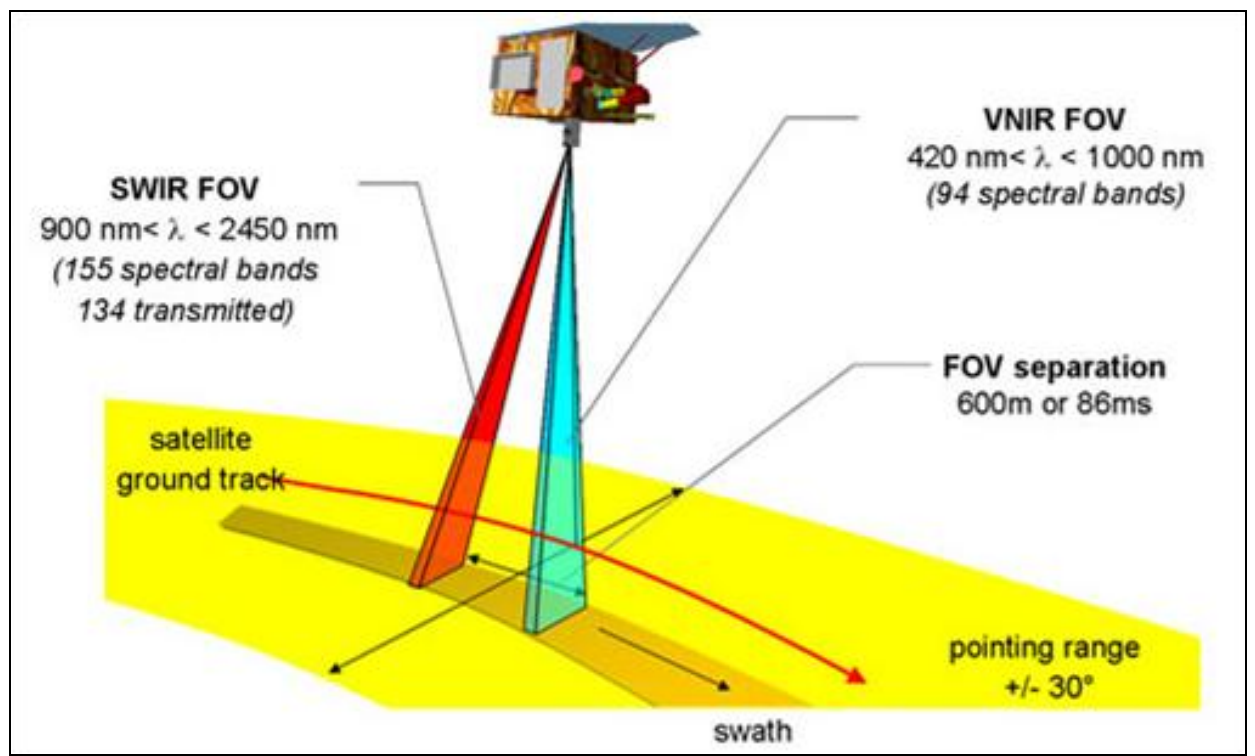

Figure 1: EnMAP overview of satellite bus and instrument data

Preparation of mission operations has some challenging aspects based on the design of the satellite bus and the type of sensor used. The bus, which is a standard bus, has restrictions concerning request of telemetry and uplink/ storage of telecommands which have to be solved by the underlying operations concept. Another difficulty is based on the fact that a hyperspectral payload is used: clouds obscure the view of this sensor on the earth's surface. Therefore operations and mission planning as a subsystem have to handle this aspect additionally. This is the first satellite mission where cloud coverage has to be taken into account in acquisition planning.

\section{EnMAP Satellite Mission Overview}

The EnMAP mission senses the surface of the Earth with a hyperspectral instrument, HSI $^{5,6}$. Main objectives are to measure and derive vital parameters of land and water sites. The Space Agency of the German Aerospace Aministration is responsible for mission management. The space segment is provided by Kayser-Threde GmbH (KT) and Orbitale Hochtechnologie Bremen-System AG (OHB System). The ground segment is prepared by the German Aerospace Center, consisting of the three parts Mission Operations Segment (MOS), the Payload Ground Segment (PGS) and the Processor, Calibration and Validation segment (PCV). The satellite is planned for launch in 2013 into a SSO orbit with an altitude of $643 \mathrm{~km}$. Planned mission duration is 5 years.

The HSI instrument is designed as a push-broom scanner covering an area of $30 \times 30 \mathrm{~m}$ per pixel at $48^{\circ}$ north nadir viewing. An area up to $1000 \mathrm{~km}$ length with a swath of $30 \mathrm{~km}$ (nadir) can be covered per orbit; maximum length per day is $5000 \mathrm{~km}$. Within the range of $5000 \mathrm{~km}$ a maximum of 50 images per day is possible. For off-nadir acquisitions the instrument can be rolled $30^{\circ}$ into both directions. Operations are controlled by TC and TM transfer via S-band uplink and payload data are received via X-band downlink.

\section{Operational Constraints}

S-band communications uses a standard uplink data rate of $4 \mathrm{kbps}$ for TC and TM is downlinked at a speed of 64 kbps. Operations of the satellite bus are driven by the main challenges:

1) Number of time tagged telecommands (TT-TC), which can be stored by the satellite

2) Request of house keeping (HK) packets

\section{A. Time Tagged Telecommand Storage}


Time tagged TC are stored by EnMAP in a memory called TT register. The bus is configured to contain $120 \mathrm{TT}$ TCs (in the basic design only 80 where planned). One limit is the TC memory of $517 \mathrm{kByte}$, another the design of the onboard software. This leads to good accommodation of "big" telecommands as needed for datatakes for example, or orbit maneuvers. In that case one TC is associated with a set of actions belonging together from a commanding point of view. On the other hand also one TT TC register is needed for "small" very atomic commands like autonomous telemetry turn on or setting of onboard min and max values.

This number of TT TC limits the daily operations as all data take and bus commanding must be covered. Other satellites offer considerably more registers, for example the missions TET, TDX or TSX offer a range between 1000 -5200 register spaces.

The difficulty here is to plan activities very carefully because only 120 register spaces are available. The mission planning system must consider this planning problem to get all commands in the TT register. For nominal but not every day business like orbit maneuvers more TT register spaces are needed for uploading e.g. the guidance list and to turn ON and OFF the thruster heaters/command the thrusters OPEN and CLOSE again etc. - this has to be considered in the planning process as well. Every command will "cost" one TT register space. It does not matter what kind of command it is. For example a whole guidance list for an ACS maneuver will take one register space as well as downloading the MIN/MAX value list. The deletion of the TTs has to be done one by one. To do this the TIDs (Telecommand IDentifier) are necessary. These are addressed to each TT before commanding and have to be remembered for deletion.

\section{B. Reception of Satellite Telemetry}

EnMAP telemetry data have to be requested via immediate command from ground; other satellite missions sent telemetry without requesting each TM packet by TC. For this bus each command triggers an answer from the satellite: it can be either a nominal telemetry packet or an error telemetry packet. A nominal telemetry packet contains the result of the execution (it can be empty but testifies the correct execution) of the command whereas an error telemetry packet indicates the error group and error code of the related command.

Dedicated commands allow retrieving telemetry data, which are sent back in the nominal telemetry packet. The two main commands are for getting the status data and for getting the current housekeeping data set. It is not possible to download all the telemetry parameters with only these two commands. Others commands are required to download very specific information e.g. attitude, reactions wheel, magnet torquer, star sensor, magnetometer or sun present status, as well as the time tagged TC list. Considering the limited uplink capability and the fact that there should be no commanding during the HK dumping (see below - Autonomous Telemetry -) it is obvious that the commanding of TCs to the S/C can only be very limited.

\section{Autonomous Telemetry:}

An autonomous telemetry feature has been implemented - the uplink S-band carrier has to be activated in order to receive telemetry corresponding to a blind acquisition pass. This feature is originally only used during S-band contact. It can be turned ON and OFF - this means to get real time TM from the S/C the autonomous telemetry has to be switched ON. Otherwise during the pass only basic TM can be monitored. The switch ON command can be time tagged and it triggers the sending of the nominal telemetry packets corresponding to housekeeping and status data. This allows to get almost all the telemetry data, except mainly additional ACS data.

If during a contact autonomous telemetry is desired, to monitor the real time telemetry, the autonomous telemetry has to be switched on. To get autonomous TM from the beginning of the pass (which is recommended to lose no time of the pass) the autonomous TM should be commanded ON at the beginning of the pass (or slightly before the pass) and has to be commanded OFF at the end of the pass with a time tagged TC. But this means there are two time tagged registers already taken for each pass. With 4 to 6 passes per day this will sum up to eight to twelve reserved TT register spaces per day needed for sending autonomous telemetry. 


\section{Dumped Housekeeping Packet}

Downloading the Housekeeping History File requires to follow a special commanding sequence. This sequence sequentially requires blocks of the file to be downloaded. The blocks are then transmitted in the nominal telemetry packet; its size is limited to 4008 bytes (including header). Such a commanding sequence implies some delays due to the network between the commanding machine and the antenna. Due to the limited bandwidth of the downlink, autonomous telemetry will be affected by the download of the Housekeeping History File. Furthermore it is necessary to take into consideration that in case of an uplink failure also the download is stopped, according to the philosophy of history telemetry file dump.

In most of the cases during the pass the autonomous TM will be turned on at the beginning of the pass to quickly check the status of the subsystems. As soon as the status is checked (as fast as possible) the dump of the housekeeping files will be triggered and the autonomous TM will be switched OFF. The switching off of the autonomous TM is necessary because otherwise the dump will most likely not be finished during the pass. On the other hand if the dump would be triggered at the beginning of the pass the TM parameters which should be monitored would be delayed for several seconds. This may cause irritation and lead to misinterpretation. Especially when the status check of the subsystems is not as expected, and an off nominal value occurs, it is absolutely necessary to react fast and see the TM in real time and without delay caused by the dump. The limited bandwidth of the downlink implies not only interaction between the autonomous telemetry and the download of the housekeeping history file, but also with the download of the Status File or even the ACS file.

\section{Cloud Coverage in EnMAP Images}

\section{EnMAP Project Aspects}

The aspect of potential cloud coverage in EnMAP images has been discussed throughout all project phases of the mission. First ideas of how to handle this problem have already been discussed during phase A but analyses of the problem has been shifted into project phase $\mathrm{B}$ with a subsequent mission planning system design during project phase C. One reason was the involvement of many different parts of the grounds segment. Beside the MOS segment also PCV and PGS contributed to the discussion. PCV is responsible for processing of raw image data and identification of clouds in satellite images (L0 processor). So PCV is the source for MOS concerning the results of image acquisition planning and resulting cloud contamination of images. L0 processor results are forwarded via PGS (taking care of data management) to MOS. Any decision of image reordering is made in PGS, setting the maximum cloud thresholds for all user of a certain category. Therefore also PGS where involved in the discussion. Last but not least also the project management as well as the principal investigator is in the loop, as all reordering of images limits the amount of acquisitions available to other users.

It turned out that discussing the different options with respect to handling of image cloud coverage should be documented in a project technical note. This TN is part of the project documentation and should be provided by for example the MPS group. Aspects from other ground segment systems should also be covered in this document. We recommended providing the $\mathrm{TN}$ to the management before any major milestone is reached - e.g. the preliminary design or critical design review. This should allow for any high level discussions (see the point above of blocking new image acquisitions by reordering of images which have been cloud contaminated).

Our TN covered the following aspects:

1) The different potential technical solutions of handling cloud coverage within the project, including the "do nothing" option

2) The design impact in all affected subsystems (for EnMAP the Data Information Management System, the L0 Processor and the Mission Planning System)

3) The cost of each solution

4) Potential benefit of each solution, as far as this can be estimated 
The fourth point is in our opinion the most challenging for each project; for EnMAP no calculations could be made for the potential benefits, but only very rough estimations about the general benefit of such an implementation. For further discussion will only include the MPS relevant aspects, therefore mainly technical details will be touched.

\section{Study of the problem and potential solutions}

We studied cloud coverage for EO satellites in a general way by review of literature and solutions suggested by other projects ${ }^{1,4}$. Clouds cover the land masses of the Earth at any point in time to about $\sim 58 \%{ }^{2}$. Images which are at least partly covered by clouds can usually only be used with average cloud coverage of maximum $\sim 15 \%{ }^{3}$. As EnMAP acquires images with an along track length of between $30-1000 \mathrm{~km}$ and an across track length of $30 \mathrm{~km}$ cloud coverage is expected to become a significant problem. Therefore we simulated the cloud coverage within the swath of the instrument. This provides a first idea how much of the area within the swath is obscured by clouds; Figure 2 shows the result for one orbit. Figure 3 shows the simulation extended to one year. Results show a coverage between $0.57-0.63 \%$ of the area that can potentially be acquired by EnMAP.

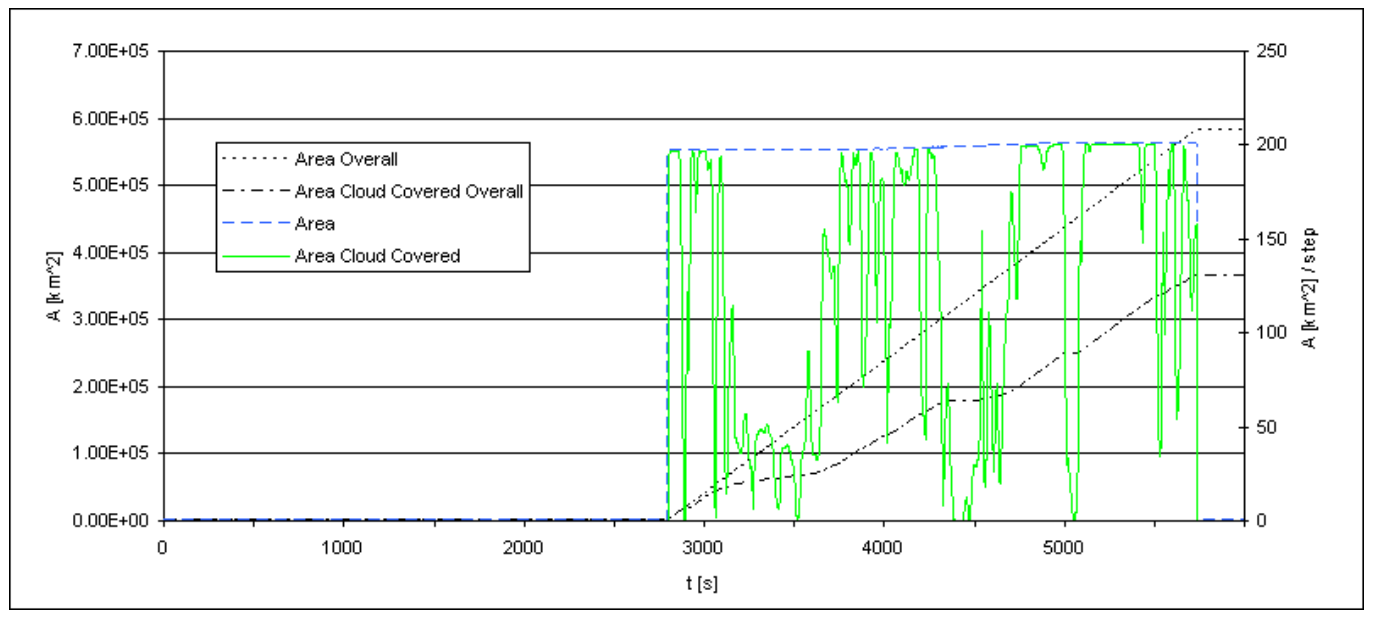

Figure 2: Cloud coverage in EnMAP image swath of one orbit: The diagram shows the area covered during one simulation step (area and area cloud covered) as well as accumulated values (area overall and area cloud covered overall) since simulation start. Simulation covers one orbit and only the sun-light fraction of the orbit (optical sensor). 


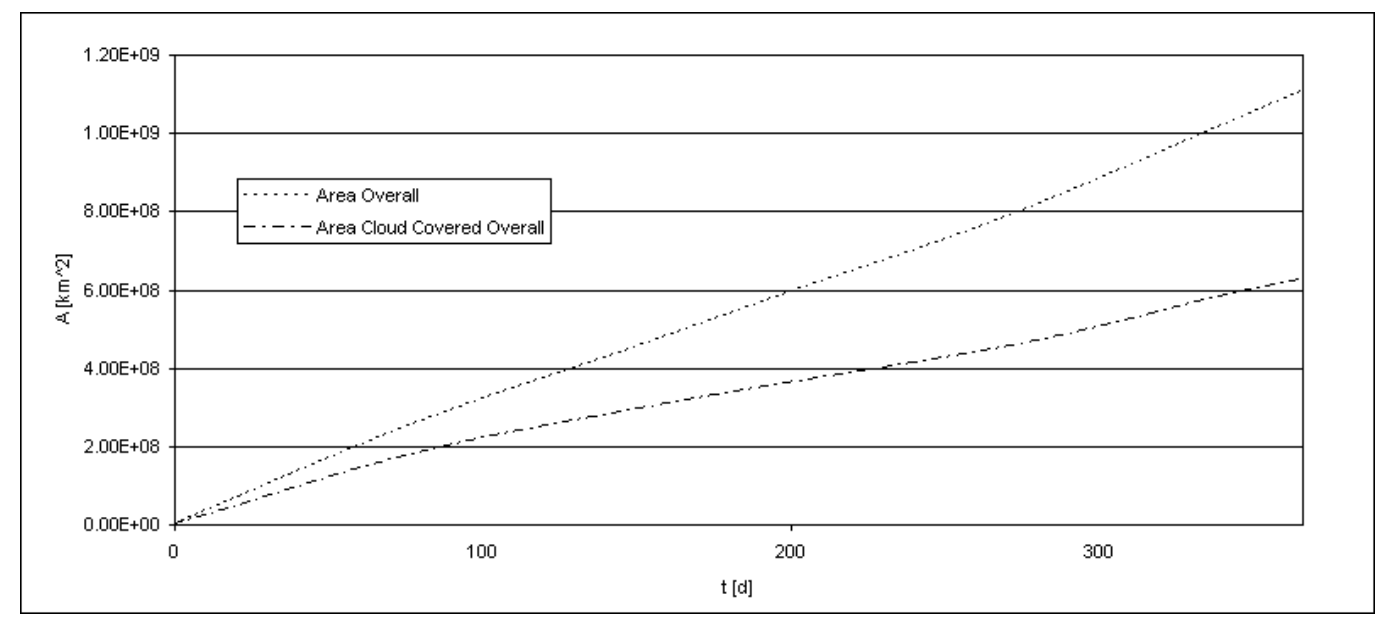

Figure 3: Cloud coverage in EnMAP image swath during one year: The diagram in Figure 3 shows accumulated values since simulation start (area overall and area cloud covered overall). The simulation covers a duration of one year.

Simulation results verify the problem significance and are in the expected range as also discussed in literature; more than $50 \%$ of the area is covered. The "do nothing" option is therefore not a really good option if the goal is maximum scientific mission utilization. This leaves to other options possible for the mission: Either to incorporate measures against cloud coverage on board the satellite or to use cloud coverage forecast during mission planning process on ground. Based on the existing spacecraft bus design we decided for the second option; a way also chosen by other EO satellite missions.

\section{E. Design of the Mission Planning System}

Design of the mission planning system requires tying to ends of a chain: On the one hand cloud coverage forecast data and on the other hand feedback of the L0 processor and resulting reorder data (provided by the Data Information and Management System (DIMS), right side of Figure 4). These two sources of information must be processed by MPS. Furthermore we tried to develop the system in a way that also other future EO missions in GSOC can use it (mission independent access).

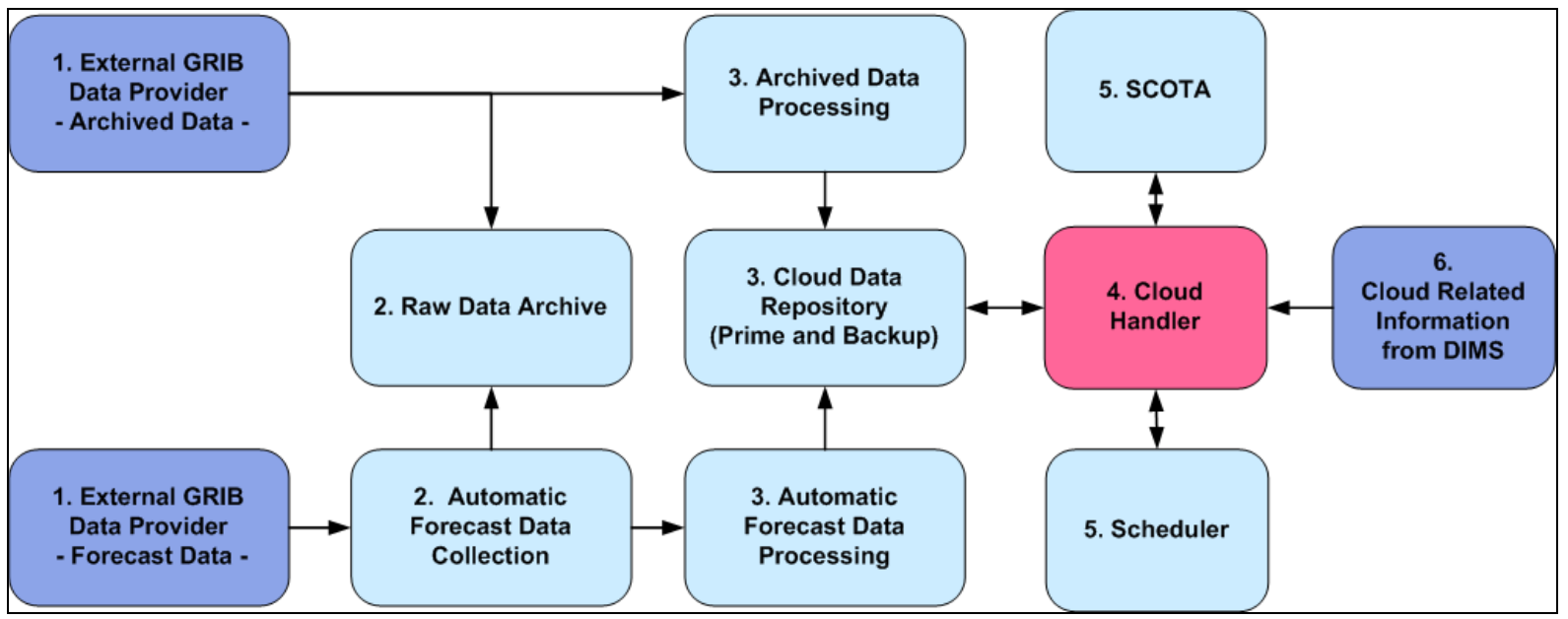

Figure 4: Cloud data processing MPS components for EnMAP Components shown are used for handling of cloud relevant information for mission planning 
1) External GRIB Data Provider: The external data provider provides archived as well as current forecast data. We decided for a numerical model (GRIB - gridded binary data) covering the whole world, although other data types and models are available.

2) Automatic Forecast Data Collection \& Raw Data Archive: Forecast data is usually provided via FTP and deleted from servers by the provider after a while, therefore automatic collection from external source and transfer as well as archival is needed. Data is stored in an archive to have the raw data available.

3) Data Processing \& Repository: Data is extracted from the original GRIB format and stored in a database. The GRIB data file contains all points of the forecast area; extraction of only single points from this file is time consuming. Data storage and retrieval from a database is faster.

4) Cloud Handler: This component is responsible for calculation of expected cloud coverage. This data is used to increase or decrease the priority of the datatake within its group of datatakes by MPS and therefore the mission specific core component.

5) SCOTA \& Scheduler: SCOTA delivers the position calculations (latitude \& longitude) for each datatake based on TLE for the orbit. This information is required for mapping cloud coverage forecasts and planned image acquisitions to an expected value for planning. The scheduler finally receives the expected cloud coverage for planning of image acquisitions.

6) DIMS: Data about results of executed image acquisitions (L0 processor results) and reordering information is received via this interface. Detailed results are used for MPS cloud coverage system tweaking.

Only one MPS component, the cloud handler, will contain EnMAP mission specific algorithms. All other components can also be used by other missions. External interfaces are the DIMS and the weather forecast service. DIMS handles user orders as well as information concerning how many times a cloud covered area should be acquired. The L0 processor analyses the X-band downlinked image information for clouds, dust and snow in the different image tiles and forwards this information through DIMS to MPS. On one hand this information is used for steering of reordering scenarios, on the other hand for MPS performance control as well as statistical purposes.

\section{Conclusion}

A compendious overview of the EnMAP satellite is given, the system, the satellite manufacturer, the ground segment and the mission operations. Operational constraints pertaining to spacecraft TM and TC are outlined. The TC architecture and procedures have been described, especially the time tagged TCs and the autonomous TM. The specifics of the autonomous TM are alluded to. On the basis of these system specifics, this spacecraft design is well suited to fulfill the mission.

The EnMAP specific mission problem of cloud coverage in images has been discussed. We recommend for similar mission to generate a project specific technical note - TN - in order to collect all operations relevant aspects of cloud coverage. This is usually not only aspects of mission planning, but also other aspects like automated cloud detection by L0 processor or management of reordering by DIMS. Furthermore such a document can be used for discussion of implementation issues together with the project management. The impact of cloud coverage on EnMAP is expected to be more than 50\% of the acquired area. A decision for a ground based system taking forecasts during image acquisition planning into account has been made. Cloud forecast relevant modules of MPS are divided into EnMAP specific and generic ones, allowing system usage also for other missions. It's important to incorporate automatic feedback of acquisition results into MPS for statistical and reordering purposes.

\section{References}

\footnotetext{
${ }^{1}$ Mandl, Dan; Miller, Jerry; Griffin, Michael; Burke, Hsiao-hua: "Validation of On-board Cloud Cover Assessment Using EO-1”, NASA Technical Report, Goddard Space Flight Center, June 9, 2003

${ }^{2}$ Rossow, W. B.; Schiffer, R. A.: “Advances in Understanding Clouds from ISCCP”. In: Bulletin of the American Meteorological Society, Volume 80, Issue 11, p. 2261-2287
} 
${ }^{3}$ Linden, N. van der; Roozekrans, H.; Som de Cerff, W.; Veen, S. van der; Beck, R.: Cloud Forecasting for Earth Observation Satellites. BCRS Report USP-2 98-20, 1999. ISBN 90- 5411-261-1

${ }^{4}$ Arvidson, T.; Gasch, J.; Goward, Samuel N.: "Landsat 7's long-term acquisition plan — an innovative approach to building a global imagery archive". Remote Sensing of Environment, Volume 78, Issues 1-2, October 2001, Pages 13-26

${ }^{5}$ Stuffler, T.; Hofer, S.; Leipold, M.; Förster, K.-P.; Sang, B.; Schubert, J.; Penné, B.; Kaufmann, H.; Müller, A.; Chlebek, C. (2009): "EnMAP - Space Segment - Instrument and Mission Parameters". In: EARSeL SIG-IS Workshop; Tel Aviv, Israel.

${ }^{6}$ Müller, R.; Bachmann, M.; Makasy, C.; Miguel, A.; Müller, A.; Neumann, A.; Palubinskas, G.; Richter, R.; Schneider, M.; Storch, T.; Walzel, T.; Kaufmann, H.; Guanter, L.; Segl, K. (2009) ENMAP - THE FUTURE HYPERSPECTRAL SATELLITE MISSION PRODUCT GENERATION. ISPRS . ISPRS Hannover Workshop 2009, High-Resolution Earth Imaging for Geospatial Information , 02.-05. Juni 2009, Hannover. ISSN 1682-1777 